\title{
ON THE DISTRIBUTION OF THE SUPREMUM FUNC- TIONAL FOR PROCESSES WITH STATIONARY INDEPENDENT INCREMENTS
}

BY

\author{
GLEN BAXTER AND M. D. DONSKER
}

1. Introduction. Let $\{x(t), 0 \leq t<\infty\}$ be a separable stochastic process with stationary independent increments whose sample functions vanish at the origin. Such a process is characterized by

$$
E\left\{e^{i \xi x(T)}\right\}=e^{T \psi(\xi)} \quad 0 \leq T<\infty
$$

where $e^{\psi(\xi)}$ is the Lévy-Khintchine representation of the characteristic function of an infinitely divisible distribution, i.e., for some real $\boldsymbol{\gamma}$ and bounded, nondecreasing function $G(x)$, with $G(-\infty)=0$,

$$
\psi(\xi)=i \gamma \xi+\int_{-\infty}^{\infty}\left(e^{i x \xi}-1-\frac{i \xi x}{1+x^{2}}\right) \frac{1+x^{2}}{x^{2}} d G(x) .
$$

Since the process is separable, the functional, $\sup _{0 \leq t \leq T} x(t)$, is measurable and it is the object of this paper to obtain its distribution function, $\sigma(\alpha, T)$ $=P\left\{\sup _{0 \leq t \leq T} x(t)<\alpha\right\}$. One of the most important processes of the type being considered for which $\sigma(\alpha, T)$ has been explicitly calculated is the Wiener process, $\psi(\xi)=-\xi^{2} / 2$, for which

$$
\sigma(\alpha, T)=\left(\frac{2}{\pi}\right)^{1 / 2} \int_{0}^{\alpha / T^{1 / 2}} e^{-s^{2} / 2} d s
$$

has been obtained by various techniques. Calculations of $\sigma(\alpha, T)$ in important special cases also have been carried out by Darling [4], Täcklind [5], and others. Although we cannot explicitly evaluate $\sigma(\alpha, T)$ in the general case, we do obtain a formula for the double Laplace transform of $\sigma(\alpha, T)$ in terms of $\psi(\xi)$.

Theorem 1. For all positive $u$ and $\lambda$,

(i) If $\psi(\xi)$ is real

$$
\begin{aligned}
u \int_{0}^{\infty} \int_{0}^{\infty} e^{-u T-\lambda \alpha} d_{\alpha} \sigma(\alpha, T) d T & \\
& =\exp \left\{\frac{1}{2 \pi} \int_{u}^{\infty} \int_{-\infty}^{\infty} \frac{\lambda}{\lambda^{2}+\xi^{2}} \frac{\psi(\xi)}{s(s-\psi(\xi))} d \xi d s\right\} .
\end{aligned}
$$

Presented to the Society, April 13, 1956; received by the editors February 21, 1956. 
(ii) If $\psi(\xi)$ is complex, and for some $\delta>0, \int_{-\delta}^{\delta}|\psi(\xi) / \xi| d \xi<\infty$,

$$
\begin{aligned}
u \int_{0}^{\infty} \int_{0}^{\infty} e^{-u T-\lambda \alpha} d_{\alpha} \sigma(\alpha, T) d T & \\
= & \exp \left\{\frac{1}{2 \pi} \int_{u}^{\infty} \int_{-\infty}^{\infty} \frac{\lambda}{\xi(\xi-i \lambda)} \frac{\psi(\xi)}{s(s-\psi(\xi))} d \xi d s\right\} .
\end{aligned}
$$

This theorem depends essentially on an elegant combinatorial identity by Spitzer [1]. (See formula (2.1) below.) Since $\int_{\mu}^{\infty} \psi(\xi) d s / s(s-\psi(\xi)$ ) $=\log (1-\psi(\xi) / u)$, in both (1.2) and (1.3) if the integration on $s$ can be brought inside the integration on $\xi$ a slightly more elegant result is obtained.

In $\S 4$ of this paper we illustrate how Theorem 1 may be used to calculate the double Laplace Transform of $\sigma(\alpha, T)$ for certain processes (certain $\psi$ 's) of interest, and in some of these how the transforms can be inverted yielding explicit formulae for $\sigma(\alpha, T)$. As an example of the latter, consider the process where $\psi(\xi)=a(\cos \xi-1)$. The sample functions, $x(t)$, of this process can be interpreted as the monetary gain in coin tossing at random times. More specifically, $x(t)$ is the sum of a random number, $N(t)$, of independent, identically distributed Bernoulli variables with distribution $P\{x=-1\}=P\{x=1\}$ $=1 / 2$, and where $N(t)$ is a sample function of the Poisson process (cf. [2, p. 398]). This process is of fundamental importance in the theory of collective risk and has been considered in detail by Täcklind [5]. In $\$ 4$ it is shown once again that for this process

$$
P\left\{\sup _{0 \leq t \leq T} x(t)<n\right\}=1-n \int_{0}^{T} e^{-a t} \frac{I_{n}(a t)}{t} d t
$$

where $J_{n}(x)$ is the Bessel function of the first kind, and where $I_{n}=i^{-n} J_{n}(i x)$.

The techniques employed in $\$ 4$ consist of various contour integration methods for evaluating the integrals on the right of (1.2) and (1.3). These methods can be applied to $\psi$ 's other than those considered in the specific examples of that section and in $\$ 5$ we prove the following theorem in this direction.

Theorem 2. Let $u$ be positive. Let $\psi(\xi)$ have an analytic continuation $\Psi(z)$ in the lower half-plane ( $\operatorname{Im} z \leq 0)$ and let $\psi(z)-u$ have only (infinitely many) simple zeros $\rho_{1}, \rho_{2}, \rho_{3}, \cdots,\left(\left|\rho_{1}\right| \leq\left|\rho_{2}\right| \leq\left|\rho_{3}\right| \leq \cdots\right)$, in the lower half-plane. Then, if $\left|\rho_{1}\right| \rightarrow \infty$ as $u \rightarrow \infty$,

$$
u \int_{0}^{\infty} \int_{0}^{\infty} e^{-u T-\lambda \alpha} d_{\alpha} \sigma(\alpha, T) d T=\frac{1}{\prod_{k=1}^{\infty}\left(1-\frac{i \lambda}{\rho_{k}}\right) e^{\sigma_{\mathbf{k}}(i \lambda)}}
$$

where the $g_{k}$ 's are appropriately chosen convergence factors depending only on $\psi(\xi)$ and $u$. (The $g_{k}$ 's are explicitly defined in (5.4) below.) 
We remark here that in case the integral

$$
\int_{-\infty}^{\infty} e^{i z \alpha} d_{\alpha} P\{x(T) \leq \alpha\}=e^{T \bar{\psi}(z)}
$$

exists for all $T>0$ and all complex $z$ with $\operatorname{Im} z \leq 0$, the condition in Theorem 2 that $\left|\rho_{1}\right| \rightarrow \infty$ as $u \rightarrow \infty$ is equivalent to the condition that for some $T>0$, $P\{x(T)>0\}>0$. Thus under (1.6), the condition on $\rho_{1}$ merely eliminates the trivial case in which $P\{x(T) \leq 0\}=1$ for all $T>0$.

2. Spitzer's identity and lemmas. Let $\left\{x_{k}\right\}$ be a sequence of independent, identically distributed random variables with partial sums $s_{k}$. Let $s_{k}^{+}=s_{k}$ if $s_{k}>0$ and 0 otherwise. For $k=1,2,3, \cdots$ let

$$
\phi_{k}(\lambda)=\int_{0}^{\infty} e^{-\lambda \alpha} d P\left\{\max _{1 \leq J^{\prime} K} s_{j}^{+}<\alpha\right\}, \quad \psi_{k}(\lambda)=\int_{0}^{\infty} e^{-\lambda \alpha} d P\left\{s_{k}^{+}<\alpha\right\},
$$

and let $\phi_{0}(\lambda) \equiv 1$. A theorem of Spitzer, [1], gives for $|t|<1$,

$$
(1-t) \sum_{k=0}^{\infty} \phi_{k}(\lambda) t^{k}=\exp \left\{-\sum_{k=1}^{\infty}\left(\frac{1-\psi_{k}(\lambda)}{k}\right) t^{k}\right\} .
$$

In addition to Spitzer's identity we will need two lemmas for the proof of Theorem 1. The first of these is a straightforward generalization of the Lévy inversion formula for characteristic functions.

Lemma 1. Let $F(\alpha)$ be a distribution function with characteristic function $\phi(\xi)$. Let $g(\alpha)$ be piecewise continuous, of bounded variation on $(-\infty, \infty)$, and let $g(\alpha)$ be in $L_{1}(-\infty, \infty)$. Then,

$$
\frac{1}{2} \int_{-\infty}^{\infty}[g(\alpha+0)+g(\alpha-0)] d F(\alpha)=\frac{1}{2 \pi} \lim _{U \rightarrow \infty} \int_{-U}^{U} \int_{-\infty}^{\infty} e^{-i \xi s} g(s) d s \phi(\xi) d \xi .
$$

The Lévy inversion formula is obtained from (2.2) by letting $g(\alpha)=1$ for $\alpha$ in a continuity interval of $F(\alpha)$ and 0 otherwise. To prove (2.2), let

$$
I_{U}=\frac{1}{2 \pi} \int_{-U}^{U}\left[\int_{-\infty}^{\infty} e^{-i \xi s} g(s) d s \int_{-\infty}^{\infty} e^{i \xi \alpha} d F(\alpha)\right] d \xi=\int_{-\infty}^{\infty} f(U, \alpha) d F(\alpha),
$$

where

$$
\begin{aligned}
f(U, \alpha) & =\frac{1}{2 \pi} \int_{-U}^{U} e^{i \xi \alpha} \int_{-\infty}^{\infty} e^{-i \xi s} g(s) d s d \xi \\
& =\frac{1}{\pi} \int_{-\infty}^{\infty} \frac{\sin U(s-\alpha)}{s-\alpha} g(s) d s .
\end{aligned}
$$

Now 


$$
\lim _{U \rightarrow \infty} \frac{1}{\pi} \int_{-\infty}^{\infty} \frac{\sin U(s-\alpha)}{s-\alpha} g(s) d s=\frac{1}{2}[g(\alpha+0)+g(\alpha-0)] .
$$

Furthermore, from dominated convergence of $f(U, \alpha)$ as $U \rightarrow \infty$ we have

$$
\lim _{U \rightarrow \infty} I_{U}=\frac{1}{2} \int_{-\infty}^{\infty}[g(\alpha+0)+g(\alpha-0)] d F(\alpha) .
$$

LemMa 2. Let $\{x(t), 0 \leq t<\infty\}$ be a separable stochastic process with stationary independent increments whose sample functions vanish at the origin. Let $x^{+}(t)=x(t)$ if the latter function is positive and 0 otherwise. Let

$$
\begin{aligned}
\phi(\lambda, T) & =\int_{0}^{\infty} e^{-\lambda \alpha} d_{\alpha} P\left\{\sup _{0 \leq t \leq T} x(t)<\alpha\right\}, \\
\phi_{N}(\lambda, T) & =\int_{0}^{\infty} e^{-\lambda \alpha} d_{\alpha} P\left\{\max _{1 \leq j \leq\left[T 2^{n}\right]} x^{+}\left(\frac{j}{2^{N}}\right)<\alpha\right\} .
\end{aligned}
$$

Then, $\lim _{N \rightarrow \infty} \phi_{N}(\lambda, T)=\phi(\lambda, T)$ uniformly for $T$ in any finite interval.

To prove Lemma 2 , we note that since $\phi_{N}(\lambda, T)$ is monotone decreasing in $N$, it will be sufficient to show $\lim _{N \rightarrow \infty} \phi_{N}(\lambda, T)=\phi(\lambda, T)$ for each $T$ in the finite interval and that $\phi(\lambda, T)$ is a continuous function of $T$.

The fact that $\lim _{N \rightarrow \infty} \phi_{N}(\lambda, T)=\phi(\lambda, T)$ for each fixed $T$ follows from the Helly-Bray Theorem, the separability of the process, and the fact that

$$
P\left\{\sup _{0 \leq t \leq T} x^{+}(t)<\alpha\right\}=P\left\{\sup _{0 \leq t \leq T} x(t)<\alpha\right\} \text {. Let } \tilde{x}(T)=\sup _{0 \leq t \leq T} x(t) .
$$

Since the process $x(t)$ has no fixed points of discontinuity we have

$$
\lim _{\Delta T \rightarrow 0}[x(T+\Delta T)-x(T)]=\lim _{\Delta T \rightarrow 0}[\tilde{x}(T+\Delta T)-\tilde{x}(T)]=0
$$

with probability one. From the continuity theorem for characteristic functions we thus obtain

$$
\lim _{\Delta T \rightarrow 0} \phi(\lambda, T+\Delta T)=\phi(\lambda, T) .
$$

3. Proof of Theorem 1. Again letting $\phi(\lambda, T)=\int_{0}^{\infty} e^{-\lambda \alpha} d_{\alpha} \sigma(\alpha, T)$, we can write

$$
\begin{aligned}
& u \int_{0}^{\infty} \int_{0}^{\infty} e^{-u T-\lambda \alpha} d_{\alpha} \sigma(\alpha, T) d T=u \int_{0}^{\infty} e^{-u T} \phi(\lambda, T) d T \\
& =\lim _{N \rightarrow \infty}\left(\frac{1-e^{-u / 2^{N}}}{1 / 2^{N}}\right) \sum_{k=0}^{\infty} \phi\left(\lambda, \frac{k}{2^{N}}\right) e^{-u k / 2^{N}} \cdot \frac{1}{2^{N}} \\
& =\lim _{N \rightarrow \infty}\left(1-e^{-u / 2^{N}}\right) \sum_{k=0}^{\infty} \phi\left(\lambda, \frac{k}{2^{N}}\right) e^{-u k / 2^{N}} .
\end{aligned}
$$


Let $x^{+}(t)=x(t)$ if $x(t) \geq 0$ and 0 otherwise. We show now that on the right side of (3.1) we can replace $\phi\left(\lambda, k / 2^{N}\right)$ by

$$
\phi_{N}\left(\lambda, \frac{k}{2^{N}}\right)=\int_{0}^{\infty} e^{-\lambda \alpha} d_{\alpha} P\left\{\max _{1 \leq j \leq k} x^{+}\left(\frac{j}{2^{N}}\right)<\alpha\right\} .
$$

To show this, let an $\epsilon>0$ be given, and let $K$ be a fixed positive integer so large that $e^{-u{ }^{1} K}<\epsilon / 4$. From Lemma 2, we have for sufficiently large $N$,

$$
\left|\phi_{N}\left(\lambda, \frac{k}{2^{N}}\right)-\phi\left(\lambda, \frac{k}{2^{N}}\right)\right| \leq \frac{\epsilon}{2} \quad k=0,1, \cdots, 2^{N+K} .
$$

Therefore, for sufficiently large $N$

$$
\begin{aligned}
\mid\left(1-e^{-u / 2^{N}}\right) \sum_{k=0}^{\infty} & {\left[\phi_{N}\left(\lambda, \frac{k}{2^{N}}\right)-\phi\left(\lambda, \frac{k}{2^{N}}\right)\right] e^{-u k / 2^{N}} \mid } \\
\leq & \left(1-e^{-u / 2^{N}}\right) \sum_{k=0}^{2^{N+K}}\left|\phi_{N}\left(\lambda, \frac{k}{2^{N}}\right)-\phi\left(\lambda, \frac{k}{2^{N}}\right)\right| e^{-u k / 2^{N}} \\
& +2\left(1-e^{-u / 2^{N}}\right) \sum_{k=2^{N+K}}^{\infty} e^{-u k / 2^{N}} \\
\leq & \frac{\epsilon}{2}+2 \cdot \frac{\epsilon}{4}=\epsilon .
\end{aligned}
$$

From (3.1) and (3.3), we obtain

$$
u \int_{0}^{\infty} e^{-u T} \phi(\lambda, T) d T=\lim _{N \rightarrow \infty}\left(1-e^{-u / 2^{N}}\right) \sum_{k=0}^{\infty} \phi_{N}\left(\lambda, \frac{k}{2^{N}}\right) e^{-u k / 2^{N}} .
$$

Let $\psi(\lambda, T)=\int_{0}^{\infty} e^{-\lambda \alpha} d_{\alpha} P\left\{x^{+}(T)<\alpha\right\}$. We now apply Spitzer's identity (2.1) to the right side of (3.4) with $x_{k}=x\left(k / 2^{N}\right)-x\left((k-1) / 2^{N}\right) k=1,2, \cdots$ and with $t=e^{-u / 2^{N}}$, obtaining

$$
u \int_{0}^{\infty} e^{-u T} \phi(\lambda, T) d T=\exp \left\{\lim _{N \rightarrow \infty} \sum_{k=1}^{\infty}\left(\frac{\psi\left(\lambda, k / 2^{N}\right)-1}{k}\right) e^{-u k / 2^{N}}\right\}
$$

Now clearly for $u>0$

$$
\begin{aligned}
\int_{u}^{\infty} \int_{0}^{\infty} e^{-s T}(\psi(\lambda, T)-1) & d T d s \\
= & \int_{u}^{\infty} \lim _{N \rightarrow \infty} \sum_{k=1}^{\infty}\left(\psi\left(\lambda, \frac{k}{2^{N}}\right)-1\right) e^{-s k / 2^{N}} d s \\
& =\lim _{N \rightarrow \infty} \int_{u}^{\infty}\left(\sum_{k=1}^{\infty}\left(\psi\left(\lambda, \frac{k}{2^{N}}\right)-1\right) e^{-s k / 2^{N}} \frac{1}{2^{N}}\right) d s \\
& =\lim _{N \rightarrow \infty} \sum_{k=1}^{\infty}\left(\frac{\psi\left(\lambda, k / 2^{N}\right)-1}{k}\right) e^{-u k / 2^{N}} .
\end{aligned}
$$


Hence, from (3.5) we get ${ }^{1}$ )

$$
u \int_{0}^{\infty} e^{-u T} \phi(\lambda, T) d T=\exp \left\{\int_{u}^{\infty} \int_{0}^{\infty} e^{-s T}(\psi(\lambda, T)-1) d T d s\right\}
$$

We now use Lemma 1 to express the integral on the right of (3.6) in terms of $\psi(\xi)$. We will have to consider separately the two cases of Theorem 1 . First of all let us note that

$$
\psi(\lambda, T)-1=\int_{0}^{\infty}\left(e^{-\lambda \alpha}-1\right) d_{\alpha} P\{x(T)<\alpha\} .
$$

Case $(i): \psi(\xi)$ real valued.

Using Lemma 1 with

$$
g(\alpha)= \begin{cases}e^{-\lambda \alpha} & \alpha \geqq 0 \\ 0 & \alpha<0\end{cases}
$$

we obtain, since $\psi(\xi)$ is real valued,

$$
\begin{aligned}
\int_{0}^{\infty} e^{-\lambda \alpha} d_{\alpha} P\{x(T)<\alpha\}-\frac{1}{2} P\{x(T) & =0\} \\
= & \lim _{U \rightarrow \infty} \frac{1}{2 \pi} \int_{-U}^{U} \frac{1}{\lambda+i \xi} e^{T \psi(\xi)} d \xi=\frac{1}{2 \pi} \int_{-\infty}^{\infty} \frac{\lambda}{\lambda^{2}+\xi^{2}} e^{T \psi(\xi)} d \xi .
\end{aligned}
$$

Also, since $\psi(\xi)$ real valued implies $x(t)$ is symmetric we have

$$
\begin{aligned}
\int_{0}^{\infty} d_{\alpha} P\{x(T)<\alpha\} & =\frac{1}{2}+\frac{1}{2} P\{x(T)=0\} \\
& =\frac{1}{2 \pi} \int_{-\infty}^{\infty} \frac{\lambda d \xi}{\lambda^{2}+\xi^{2}}+\frac{1}{2} P\{x(T)=0\} .
\end{aligned}
$$

From (3.7), (3.8) and (3.9) we get

and therefore

$$
\psi(\lambda, T)-1=\frac{1}{2 \pi} \int_{-\infty}^{\infty} \frac{\lambda}{\lambda^{2}+\xi^{2}}\left(e^{T \psi(\xi)}-1\right) d \xi,
$$

$$
\begin{aligned}
\int_{0}^{\infty} e^{-s T}(\psi(\lambda, T)-1) d T & =\frac{1}{2 \pi} \int_{-\infty}^{\infty} \frac{\lambda}{\lambda^{2}+\xi^{2}}\left[\frac{1}{s-\psi(\xi)}-\frac{1}{s}\right] d \xi \\
& =\frac{1}{2 \pi} \int_{-\infty}^{\infty} \frac{\lambda}{\lambda^{2}+\xi^{2}} \frac{\psi(\xi)}{s(s-\psi(\xi))} d \xi .
\end{aligned}
$$

(1) Formula (3.6) was obtained by the authors after seeing a statement of (2.1) in Spitzer, Bull. Amer. Math. Soc. Abstract 61-6-819, November 1955. Subsequently they were informed by Spitzer that he is aware of a continuous analogue of (2.1). 
Finally, from (3.6) and (3.10) we have (i) of Theorem 1,

$$
u \int_{0}^{\infty} e^{-u T} \phi(\lambda, T) d T=\exp \left\{\frac{1}{2 \pi} \int_{u}^{\infty} \int_{-\infty}^{\infty} \frac{\lambda}{\lambda^{2}+\xi^{2}} \frac{\psi(\xi)}{s(s-\psi(\xi))} d \xi d s\right\} .
$$

Case (ii). $\psi(\xi)$ complex, and for some $\delta>0, \int_{-\delta}^{\delta}|\psi(\xi) / \xi| d \xi<\infty$.

Again we apply Lemma 1 , but this time with

$$
g_{\epsilon}(\alpha)=\left\{\begin{array}{lr}
e^{-\lambda \alpha}-e^{-\epsilon \alpha} & \alpha \geq 0, \\
0 & \alpha<0
\end{array}\right.
$$

so that $(\epsilon>0)$

$$
\begin{aligned}
\int_{0}^{\infty} & \left(e^{-\lambda \alpha}-e^{-\epsilon \alpha}\right) d_{\alpha} P\{x(T)<\alpha\} \\
& =\lim _{U \rightarrow \infty} \frac{1}{2 \pi} \int_{-U}^{U}\left(\frac{1}{\lambda+i \xi}-\frac{1}{\epsilon+i \xi}\right) e^{T \psi(\xi)} d \xi \\
& =\frac{1}{2 \pi} \int_{-\infty}^{\infty} \frac{\lambda-\epsilon}{(\xi-i \lambda)(\xi-i \epsilon)}\left(e^{T \psi(\xi)}-1\right) d \xi .
\end{aligned}
$$

Hence,

$$
\begin{aligned}
\int_{0}^{\infty} e^{-\delta T} \int_{0}^{\infty} & \left(e^{-\lambda \alpha}-e^{-\epsilon \alpha}\right) d_{\alpha} P\{x(T)<\alpha\} d T \\
& =\frac{1}{2 \pi} \int_{-\infty}^{\infty} \frac{(\lambda-\epsilon)}{(\xi-i \lambda)(\xi-i \epsilon)} \frac{\psi(\xi)}{s(s-\psi(\xi))} d \xi .
\end{aligned}
$$

Now, for $|\xi|<\delta$ and since the real part of $\psi(\xi)$ is negative, we have for sufficiently small $\epsilon$,

$$
\left|\frac{\lambda-\epsilon}{(\xi-i \lambda)(\xi-i \epsilon)} \frac{\psi(\xi)}{s(s-\psi(\xi))}\right| \leq \frac{1}{s^{2}}\left|\frac{\psi(\xi)}{\xi}\right|
$$

and for $|\xi|>\delta$

$$
\left|\frac{\lambda-\epsilon}{(\xi-i \lambda)(\xi-i \epsilon)} \frac{\psi(\xi)}{s(s-\psi(\xi))}\right| \leq \frac{2}{s} \frac{\lambda}{\xi^{2}} .
$$

Thus, since we are assuming $\int_{-\delta}^{\delta}|\psi(\xi) / \xi| d \xi<\infty$, we obtain from (3.12) and from dominated convergence on taking the limit as $\epsilon \rightarrow 0$,

$$
\begin{aligned}
\int_{0}^{\infty} e^{-s T} \int_{0}^{\infty}\left(e^{-\lambda \alpha}-1\right) d_{\alpha} P\{x(T) & <\alpha\} d T \\
& =\frac{1}{2 \pi} \int_{-\infty}^{\infty} \frac{\lambda}{\xi(\xi-i \lambda)} \frac{\psi(\xi)}{s(s-\psi(\xi))} d \xi
\end{aligned}
$$


Finally, from (3.6) and (3.15) we have (ii) of Theorem 1,

$$
u \int_{0}^{\infty} e^{-u T} \phi(\lambda, T) d T=\exp \left\{\frac{1}{2 \pi} \int_{u}^{\infty} \int_{-\infty}^{\infty} \frac{\lambda}{\xi(\xi-i \lambda)} \frac{\psi(\xi)}{s(s-\psi(\xi))} d \xi d s\right\} .
$$

4. Examples. In this section we consider four examples in which we use Theorem 1 to calculate the double Laplace transform of $\sigma(\alpha, T)$. In the first three of these examples the $\psi$ 's involved are all entire functions, and the same sort of contour integration method applies in each of the three cases. The method is quite general and applies to many other processes than those considered. The fourth example concerns certain symmetric stable processes, and the calculation of the integral on the right of (1.3) requires a special technique.

For the special case where $\psi(\xi)$ has an analytic continuation $\psi(z)$ into the lower half-plane $(\operatorname{Im} z \leq 0)$ we start by deriving an identity useful in what follows. Let $\lambda>0$ be fixed, let $s>0$, and let $\Gamma$ be a simple closed curve in the lower half-plane. Moreover, suppose $\bar{\psi}(z) \neq s$ for all $z$ on $\Gamma$ and let $\bar{\psi}(z)-s$ have only simple zeros at points $\rho_{1}, \rho_{2}, \cdots, \rho_{n}$ inside $\Gamma$. We may then write

$$
1-\frac{\bar{\psi}(z)}{s}=e^{h(s, z)} \prod_{k=1}^{n}\left(1-\frac{z}{\rho_{k}}\right)
$$

for all $z$ inside or on $\Gamma$ and where $h(s, z)$ is analytic inside and on $\Gamma$. Taking the logarithmic derivative of both sides of (4.1) we obtain

$$
\frac{\bar{\psi}(s)}{s(s-\bar{\psi}(s))}=\frac{\partial}{\partial s}[h(s, z)]+\sum_{k=1}^{n} \frac{z}{\rho_{k}\left(\rho_{k}-z\right)} \cdot \frac{d \rho_{k}}{d s} .
$$

Multiplying both sides of (4.2) by $(2 \pi)^{-1}(\lambda / z(z-i \lambda))$ and integrating both sides around $\Gamma$ in a clockwise direction we obtain the desired identity:

$$
\frac{1}{2 \pi} \int_{\Gamma} \frac{\lambda}{z(z-i \lambda)} \frac{\bar{\psi}(s)}{s(s-\bar{\psi}(z))} d z=\sum_{k=1}^{n} \frac{i \lambda}{\rho_{k}\left(\rho_{k}-i \lambda\right)} \cdot \frac{d \rho_{k}}{d s} .
$$

EXample 1. The Gaussian case. Suppose $\psi(\xi)=i \gamma \xi-\sigma^{2} \xi^{2} / 2$. Let $\Gamma_{n}$ consist of the semi-circle $|z|=n(\operatorname{Im} z \leq 0)$, together with the portion of the real axis $-n \leq \operatorname{Re} z \leq n$.

For sufficiently large $n$, there is exactly one zero of $\psi(z)-s$ within $\Gamma_{n}$, namely, $\rho_{s}=i\left(\gamma / \sigma^{2}-\left(\left(\gamma / \sigma^{2}\right)^{2}+2 s / \sigma^{2}\right)^{1 / 2}\right)$. Applying (4.3) and noting that as $n$ becomes infinite the part of the contour integral over the semi-circle tends to zero, we get

$$
\frac{1}{2 \pi} \int_{-\infty}^{\infty} \frac{\lambda}{\xi(\xi-i \lambda)} \frac{\psi(\xi)}{s(s-\psi(\xi))} d \xi=\frac{i \lambda}{\rho_{s}\left(\rho_{s}-i \lambda\right)} \frac{d \rho_{s}}{d s},
$$

which becomes on integrating with respect to $s$ 


$$
\frac{1}{2 \pi} \int_{u}^{\infty} \int_{-\infty}^{\infty} \frac{\lambda}{\xi(\xi-i \lambda)} \frac{\psi(\xi)}{s(s-\psi(\xi))} d \xi d s=-\log \left(1-\frac{i \lambda}{\rho_{u}}\right)
$$

Thus, from (1.3)

$$
\int_{0}^{\infty} \int_{0}^{\infty} e^{-u T-\lambda \alpha} d_{\alpha} \sigma(\alpha, T) d T=\frac{\rho_{u}}{u\left(\rho_{u}-i \lambda\right)}
$$

$$
=\frac{\left(\left(\frac{\gamma}{\sigma^{2}}\right)^{2}+\frac{2 u}{\sigma^{2}}\right)^{1 / 2}-\frac{\gamma}{\sigma^{2}}}{u\left[\lambda+\left(\left(\frac{\gamma}{\sigma^{2}}\right)^{2}+\frac{2 u}{\sigma^{2}}\right)^{1 / 2}-\frac{\gamma}{\sigma^{2}}\right]} .
$$

An easy inversion of (4.6) with respect to $u$ and $\lambda$ yie!ds the density function

$$
(4.7) \frac{\partial \sigma(\alpha, T)}{\partial \alpha}=\left\{\begin{array}{rr}
\left(\frac{2}{\pi T \sigma^{2}}\right)^{1 / 2} \exp \left(-\frac{1}{2 \sigma^{2}}\left(\frac{\alpha}{T^{1 / 2}}+\gamma T^{1 / 2}\right)^{2}\right) \\
-\frac{\gamma}{\sigma^{2}} e^{2 \alpha \gamma / \sigma^{2}} \operatorname{erfc}\left[\frac{1}{\left(2 \sigma^{2}\right)^{1 / 2}}\left(\frac{\alpha}{T^{1 / 2}}+\gamma T^{1 / 2}\right)\right] & \alpha \geq 0, \\
0 & \alpha<0 .
\end{array}\right.
$$

We note that in the special case $\sigma^{2}=1, \gamma=0,(4.7)$ reduces to the density function for the sup on the Wiener process in (1.1).

EXAMPLE 2. Coin tossing at random times. We now consider the process for which $\psi(\xi)=a(\cos \xi-1)(a>0)$. As mentioned in the introduction, the sample function $x(t)$ of this process can be interpreted as the sum of a random number $N(t)$ of independent, identically distributed Bernoulli variables with distributions $P\{x=1\}=P\{x=-1\}=1 / 2$, where $N(t)$ is a sample function of the Poisson process.

To evaluate the integral on the right of (1.3) for this process, we make use again of (4.3) where we let $\Gamma_{n}$ be the square two sides of which are $z= \pm(2 n+1) \pi+i y$ with $-(4 n+2) \pi \leq y \leq 0$. Contained within $\Gamma_{n}$ are $2 n+1$ simple zeros of $\psi(z)-s$ namely $\rho_{k}=2 k \pi-i \cosh ^{-1}((s / a)+1),-n \leq k \leq n$.

Note that

$$
d \rho_{k} / d s=d \rho_{0} / d s, \quad-n \leq k \leq n .
$$

If we let $\sigma_{s}=\cosh ^{-1}((s / a)+1)$, we get from (4.3)

$$
\begin{aligned}
\frac{1}{2 \pi} \int_{\Gamma_{n}} \frac{\lambda}{z(z-i \lambda)} \frac{\psi(z)}{s(s-\psi(z))} d z \\
\quad=i \frac{d \sigma_{s}}{d s}\left\{\sum_{k=-n}^{n} \frac{1}{i\left(\sigma_{s}+\lambda\right)-2 k \pi}-\sum_{k=-n}^{n} \frac{1}{i \sigma_{s}-2 k \pi}\right\} .
\end{aligned}
$$


It is easy to see that for $n$ sufficiently large $|\psi(z) / s(s-\psi(z))|$ is uniformly bounded in $z$ and $n$ over the curve $\Gamma_{n}$. Thus as $n$ becomes infinite in (4.8) we obtain

$$
\begin{aligned}
& \frac{1}{2 \pi} \int_{-\infty}^{\infty} \frac{\lambda}{\xi(\xi-i \lambda)} \\
& \quad=\frac{\psi(\xi)}{s(s-\psi(\xi))} d \xi \\
& \quad=i \frac{d \sigma_{s}}{d s}\left\{\lim _{n \rightarrow \infty} \sum_{k=-n}^{n} \frac{1}{i\left(\sigma_{s}+\lambda\right)-2 k \pi}-\lim _{n \rightarrow \infty} \sum_{k=-n}^{n} \frac{1}{i \sigma_{s}-2 k \pi}\right\} \\
& \quad=\frac{1}{2}\left\{\operatorname{coth}\left(\frac{\sigma_{s}+\lambda}{2}\right)-\operatorname{coth} \frac{\sigma_{s}}{2}\right\} \frac{d \sigma_{s}}{d s} .
\end{aligned}
$$

Hence, integrating both sides of (4.9) on $s$ from $u$ to $\infty$,

$$
\frac{1}{2 \pi} \int_{u}^{\infty} \int_{-\infty}^{\infty} \frac{\lambda}{\xi(\xi-i \lambda)} \frac{\psi(\xi)}{s(s-\psi(\xi))} d \xi d s=\log \left(\frac{e^{\lambda / 2} \sinh \left(\sigma_{u} / 2\right)}{\sinh \left(\left(\sigma_{u}+\lambda\right) / 2\right)}\right)
$$

From (1.3) we have finally,

$$
\begin{aligned}
\int_{0}^{\infty} \int_{0}^{\infty} e^{-u T-\lambda \alpha} d_{\alpha} P\left\{\sup _{0 \leq t \leq T} x(t)<\alpha\right\} d T & =\frac{1}{u} \frac{e^{\lambda / 2} \sinh \left(\sigma_{u} / 2\right)}{\sinh \left(\left(\sigma_{u}+\lambda / 2\right)\right)} \\
& =\frac{1}{u}\left(1-e^{-\sigma_{u}}\right) \sum_{k=0}^{\infty} e^{-k \sigma_{u}-k \lambda}
\end{aligned}
$$

Inverting first with respect to $\lambda$ we find that the distribution function $P\left\{\sup _{0 \leq t \leq T} x(t)<\alpha\right\}$ is a step function of $\alpha$ with jumps only at non-negative integral values of $\alpha$. Moreover, the value of this distribution function when $\alpha=n$ as a function of $T$ is found by inverting with respect to $u$ the function,

$$
\begin{aligned}
& \frac{1}{u}\left(1-e^{-\sigma_{u}}\right) \sum_{k=0}^{n-1} e^{-k \sigma_{u}} \\
& \quad=\frac{1}{u} e^{-n \sigma_{u}}=\frac{1}{u}-\frac{1}{u}\left[-\left(\left(\frac{u}{a}+1\right)^{2}-1\right)^{1 / 2}+\left(\frac{u}{a}+1\right)\right]^{n} .
\end{aligned}
$$

We thus obtain for each positive integer $n$

$$
P\left\{\sup _{0 \leq t \leq T} x(t)<n\right\}=1-n \int_{0}^{T} e^{-a t} \frac{I_{n}(a t)}{t} d t
$$

where $J_{n}(x)$ is the Bessel function of the first kind, and where $I_{n}=i^{-n} J_{n}(i x)$.

One can also consider $\left({ }^{2}\right)$ the more general situation where

$$
\psi(\xi)=a\left[e^{i \xi} \sum_{k=0}^{\infty} p_{k} e^{-i \xi k}-1\right]
$$

(2) The referee kindly suggested this generalization of Example 2. 
with $p_{k} \geq 0, p_{0}>0, a>0$, and $\sum_{k=0}^{\infty} p_{k}=1$. If we let $f(\xi)=a\left(p_{0} e^{i \xi}-1\right)-s$ and $g(\xi)=a e^{i \xi} \sum_{k=1}^{\infty} p_{k} e^{-i \xi k}$ we easily see that on the boundary of the region $\Gamma_{k}:-(2 k+1) \pi \leq x \leq(2 k+1) \pi,-\log \left((2 a+s) / a p_{0}\right) \leq y \leq 0,|f(\xi)|>|g(\xi)|$. By Rouche's Theorem $f(\xi)$ and $f(\xi)+g(\xi)=\psi(\xi)-s$ have the same number of zeros within $\Gamma_{k}$. Since, clearly, $f(\xi)$ has exactly $2 k+1$ zeros in $\Gamma_{k}, \psi(\xi)-s$ has $2 k+1$ zeros in $\Gamma_{k}$ located at $z_{k}=2 k \pi-i \sigma_{s}$ where

$$
\psi\left(-i \sigma_{s}\right)=s=a\left[e^{\sigma_{*}} \sum_{k=0}^{\infty} p_{k} e^{-\sigma_{s} k}-1\right] .
$$

Thus, equation (4.10) continues to hold in this more general case.

Example 3. The Poisson process. In this example we consider a modified Poisson process for which $\psi(\xi)=-i \gamma \xi+a\left(e^{i \xi}-1\right)$, with $\gamma>a>0$. The evaluation of the integral on the right in (1.3) is particularly easy in this example since $\psi(z)-s$ has only one zero in the upper half-plane and $1 /(s-\psi(z))$ is bounded for $|z|>R$, Im $z \geq 0$ for sufficiently large $R$. In fact, letting

$$
\psi\left(i y_{s}\right)=s=\gamma y_{s}+a\left(e^{-y_{s}}-1\right)
$$

we obtain by the obvious contour integration (in the upper half-plane)

$$
\frac{1}{2 \pi} \int_{-\infty}^{\infty} \frac{\lambda}{\xi(\xi-i \lambda)} \frac{\psi(\xi)}{s(s-\psi(\xi))} d \xi=\frac{\psi(i \lambda)}{s(s-\psi(i \lambda))}-\frac{\lambda}{y_{s}\left(y_{s}-\lambda\right)} \frac{d y_{s}}{d s}
$$

Integrating both sides of (4.13) with respect to $s$ from $u$ to $\infty$ and using (1.3) we get

$$
u \int_{0}^{\infty} \int_{0}^{\infty} e^{-u T-\lambda \alpha} d_{\alpha} \sigma(\alpha, T) d T=\frac{1-\lambda / y_{u}}{1-\psi(i \lambda) / u} .
$$

We are unable to invert (4.14) to get $\sigma(\alpha, T)$. However, we are able to find explicitly $P\left\{\sup _{0 \leq t \leq \infty} x(t)<\alpha\right\}$. In terms of the standard Poisson process $\{y(t), 0 \leq t<\infty\},\left(\psi(\xi)=a\left(e^{i \xi}-1\right)\right)$, this is equivalent to an evaluation of the probability $P\{y(t)<\alpha+\gamma t$ for all $t\}$. We find this probability from (4.14) as follows:

$$
\begin{aligned}
\int_{0}^{\infty} e^{-\lambda \alpha} d_{\alpha} P\left\{\sup _{0 \leq i<\infty} x(t)<\alpha\right\} & =\lim _{u \rightarrow \infty} u \int_{0}^{\infty} \int_{0}^{\infty} e^{-u T-\lambda \alpha} d_{\alpha} \sigma(\alpha, T) d T \\
& =\left(\lim _{u \rightarrow 0} \frac{u}{y_{u}}\right) \frac{\lambda}{\psi(i \lambda)} \\
& =\left\{\begin{array}{cc}
(\gamma-a) \frac{\lambda}{\gamma \lambda+a\left(e^{-\lambda}-1\right)} & \gamma \geq a, \\
0 & \gamma<a .
\end{array}\right.
\end{aligned}
$$

Thus for the Poisson process $\{y(t), 0 \leq t<\infty\}$ with parameter $a$, we have for $\gamma>a$ 


$$
\begin{aligned}
& P\{y(t)<\alpha+\gamma t, 0 \leq t<\infty\} \\
&=\left\{\begin{array}{cc}
\left(1-\frac{a}{\gamma}\right) \sum_{k=0}^{[\alpha]}\left(\frac{a}{\gamma}\right)^{k} \frac{(\alpha-k)^{k}}{k !} e^{(a / \gamma)(\alpha-k)} & \alpha>0, \\
0 & \alpha \leq 0 .
\end{array}\right.
\end{aligned}
$$

In particular,

$$
P\{y(t) \leq \gamma t, 0 \leq t<\infty\}=1-\frac{a}{\gamma} .
$$

For $\left(^{3}\right)$ a bounded nondecreasing function $F(x)$, let $f(\xi)=-i \gamma \xi-\int_{0}^{\infty} d F(x)-s$ and let $g(\xi)=\int_{0}^{\infty} e^{i \xi x} d F(x)$. It is easy to verify that on the boundary of the region $R:-(1 / \gamma)\left(\int_{0}^{\infty} d F(x)+1\right) \leq \operatorname{Re} z \leq(1 / \gamma)\left(\int_{0}^{\infty} d F(x)+1\right), 0 \leq \operatorname{Im} z \leq(1 / \gamma)$ $\left(s+1+2 \int_{0}^{\infty} d F(x)\right),|f(\xi)|>|g(\xi)|$. From Rouche's Theorem we have $f(\xi)$ and $f(\xi)+g(\xi)=\psi(\xi)-s$ have the same number of zeros inside $R$. Since, clearly, $f(\xi)$ has exactly one zero inside $R, \psi(\xi)-s$ also has one zero inside $R$, namely at $i y$, where

$$
\gamma y_{s}+\int_{0}^{\infty}\left(e^{-x y_{s}}-1\right) d F(x)=s .
$$

It is now clear that (4.13) still applies as does (4.14). Equation (4.14) in this case becomes a formula of importance in the theory of collective risk and was found by different methods by Arfwedson [6, equation 46].

EXAmple $4\left({ }^{4}\right)$. Certain symmetric stable processes. It is worthwhile to note that the right side of (1.3) can be evaluated for cases in which $\psi(\xi)$ is not analytic as was the case in the previous three examples. Consider $\psi(\xi)$ $=-\gamma|\xi|^{\alpha}$ with $\gamma>0,0<\alpha \leq 2$ and where $\alpha=2 m /(2 n+1)$, with $m$ and $n$ integers. Now, for $\alpha=2 m /(2 n+1)$

$$
\frac{1}{\pi} \int_{0}^{\infty} \frac{\lambda}{\lambda^{2}+\xi^{2}} \frac{1}{s+\gamma \xi^{\alpha}} d \xi=\frac{1}{2 \pi} \int_{-\infty}^{\infty} \frac{\lambda}{\xi(\xi-i \lambda)} \frac{-\gamma|\xi|^{\alpha}}{s\left(s+\gamma|\xi|^{\alpha}\right)} d \xi+\frac{1}{2 s}
$$

In the integral on the left of (4.16), let $\tau=\xi^{2 /(2 n+1)}$ and we get

$$
\frac{1}{\pi} \int_{0}^{\infty} \frac{\lambda}{\left(\tau^{2 n+1}+\lambda^{2}\right)} \cdot \frac{1}{s+\gamma \tau^{m}}\left(n+\frac{1}{2}\right) \tau^{n-1 / 2} d \tau \equiv \frac{1}{\pi} \int_{0}^{\infty} R(\tau) \tau^{1 / 2} d \tau
$$

where $R(\tau)=\left(\lambda /\left(\tau^{2 n+1}+\lambda^{2}\right)\right)\left(1 /\left(s+\gamma \tau^{m}\right)\right)(n+1 / 2) \tau^{n+1}$ is a rational function of $\tau$ with a pole of order at most one at the origin and with a zero of order at least two at infinity. By a standard contour integration (cf. [3, p. 130]) we find the value of the integral in (4.17) to be $\pi i$ times the sum of the residues of

(3) The referee pointed out this generalization of Example 3 has application in the theory of collective risk.

(4) The authors are indebted to R. H. Cameron for suggesting the method of calculation used in Example 4. 
$R(\tau) \tau^{1 / 2}$ in the plane. Now $R(\tau) \tau^{1 / 2}$ has simple poles at the points

$$
\begin{array}{lr}
\rho_{k}=\left(\frac{s}{\gamma}\right)^{1 / m} e^{\pi i / m} e^{2 k \pi i / m} & k=0,1,2, \cdots, m-1, \\
\sigma_{k}=\lambda^{2 /(2 n+1)} e^{\pi i /(2 n+1)} e^{2 k \pi i /(2 n+1)} & k=0,1,2, \cdots, 2 n
\end{array}
$$

with residues, respectively,

$$
\begin{array}{lr}
\frac{\lambda}{\rho_{k}^{2 n+1}(s)+\lambda^{2}} \frac{d}{d s}\left(\rho_{k}^{n+1 / 2}(s)\right) & k=0,1,2, \cdots, m-1, \\
\frac{1}{2} \cdot \frac{(-1)^{k}}{s+\gamma \sigma_{k}^{m}(s)} & k=0,1,2, \cdots, 2 n .
\end{array}
$$

Thus, from (4.16), (4.17), and integrating on $s$

$$
\begin{array}{r}
\frac{1}{2 \pi} \int_{u}^{\infty} \int_{-\infty}^{\infty} \frac{\lambda}{\xi(\xi-i \lambda)} \frac{-\gamma|\xi|^{\alpha}}{s\left(s+\gamma|\xi|^{\alpha}\right)} d \xi=-\sum_{k=0}^{m-1} \frac{1}{2} \log \left(\frac{\rho_{k}^{n+1 / 2}(u)-i \lambda}{\rho_{k}^{n+1 / 2}(u)+i \lambda}\right) \\
-\sum_{k=0}^{2 n} \frac{(-1)^{k}}{2} \log \left(u+\gamma \sigma_{k}^{m}(u)\right)+\frac{1}{2} \log u
\end{array}
$$

so that

$$
\begin{aligned}
& \int_{0}^{\infty} \int_{0}^{\infty} e^{-u T-\lambda \alpha} d_{\alpha} \sigma(\alpha, T) d T \\
& \quad=\frac{1}{\mu^{1 / 2}}\left(\prod_{k=0}^{m-1} \frac{\rho_{k}^{n+1 / 2}(u)+i \lambda}{\rho_{k}^{n+1 / 2}(u)-i \lambda}\right)^{1 / 2}\left(\frac{\prod_{k=0}^{n-1}\left(u+\gamma \sigma_{2 k+1}^{m}(u)\right)}{\prod_{k=0}^{n}\left(u+\gamma \sigma_{2 l i}^{m}(u)\right)}\right)^{1 / 2}
\end{aligned}
$$

with $\rho_{k}, \sigma_{k}$ given in (4.18) and $\alpha=2 m /(2 n+1)$.

5. Proof of Theorem 2. Let $s$ be positive, let $\lambda>0$ be fixed, and let $\tilde{\rho}_{1}, \tilde{\rho}_{2}, \cdots\left(\left|\tilde{\rho}_{1}\right| \leq\left|\tilde{\rho}_{2}\right| \leq \cdots\right)$ be the simple zeros of $\Psi(z)-s$ in the lower half-plane. Select a sequence of simple closed curves $\left\{\Gamma_{n}\right\}$ having the following properties:

(i) $\Gamma_{n}$ consists in part of the real axis between $-n$ and $n$,

(ii) $\Gamma_{n}$ lies entirely in the lower half-plane $(\operatorname{Im} z \leq 0)$,

(iii) $\Gamma_{n}$ passes through none of the $\tilde{\rho}_{k}$ 's but encloses $\tilde{\rho}_{1}, \tilde{\rho}_{2}, \cdots, \tilde{\rho}_{n}$.

We define $\Gamma_{n}^{\prime}$ to be that part of $\Gamma_{n}$ excluding the real axis from $-n$ to $n$. Note that $\Gamma_{n}$ and $\Gamma_{n}^{\prime}$ are both functions of $s$. By Formula (4.3) with $\lambda>0$

$$
\frac{1}{2 \pi} \int_{\Gamma_{n}} \frac{\lambda}{z(z-i \lambda)} \frac{\bar{\psi}(z)}{s(s-\bar{\psi}(z))} d z=\sum_{k=1}^{n}\left(\frac{1}{\tilde{\rho}_{k}-i \lambda}-\frac{1}{\tilde{\rho}_{k}}\right) \frac{d \tilde{\rho}_{k}}{d s}
$$


the integral around $\Gamma_{n}$ being taken in a clockwise sense. Moreover, $\left|\tilde{\rho}_{k}\right| \rightarrow \infty$ as $s \rightarrow \infty$ since $\left|\tilde{\rho}_{1}\right| \rightarrow \infty$ as $s \rightarrow \infty$. Thus,

$$
\frac{1}{2 \pi} \int_{u}^{\infty} \int_{\Gamma_{n}} \frac{\lambda}{z(z-i \lambda)} \frac{\bar{\psi}(z)}{s(s-\bar{\psi}(z)} d z d s=-\sum_{k=1}^{n} \log \left(1-\frac{i \lambda}{\tilde{\rho}_{k}}\right)
$$

principal values for the logarithm being used throughout. Now define for Im $a>0$,

$$
\begin{aligned}
g_{1}(a)= & \frac{1}{2 \pi} \int_{u}^{\infty} \int_{\Gamma^{\prime}{ }_{1}} \frac{\lambda}{z(z-a)} \frac{\bar{\psi}(z)}{s(s-\bar{\psi}(z))} d z d s, \\
g_{k}(a)= & \frac{1}{2 \pi} \int_{u}^{\infty} \int_{\Gamma^{\prime}{ }_{k}} \frac{\lambda}{z(z-a)} \frac{\bar{\psi}(z)}{s(s-\bar{\psi}(z))} d z d s, \\
& -\frac{1}{2 \pi} \int_{u}^{\infty} \int_{\Gamma^{\prime} k_{k-1}} \frac{\lambda}{z(z-a)} \frac{\bar{\psi}(z)}{s(s-\bar{\psi}(z))} d z d s
\end{aligned}
$$

the integrals being taken around $\Gamma_{k}^{\prime}$ in a clockwise sense. We call attention to the fact that the $g_{k}(a)$ 's as defined in (5.4) are actually independent of the particular sequence $\left\{\Gamma_{n}\right\}$ chosen, and they depend only on $\psi(\xi)$ and $s$. By Cauchy's theorem any other sequence of $\Gamma_{n}$ which satisfy (5.1) yield the same functions $g_{k}(a)$. Relation (5.3) may now be written

$$
\frac{1}{2 \pi} \int_{-n}^{n} \frac{\lambda}{\xi(\xi-i \lambda)} \log \left(1-\frac{\psi(\xi)}{u}\right) d \xi=-\sum_{k=1}^{n} \log \left(1-\frac{i \lambda}{\rho_{k}}\right)+g_{k}(i \lambda)
$$

Exponentiating and passing to the limit on $n$ yields the result of Theorem 2

$$
u \int_{0}^{\infty} \int_{0}^{\infty} e^{-u T-\lambda \alpha} d_{\alpha} \sigma(\alpha, T) d T=\frac{1}{\prod_{k=1}^{\infty}\left(1-\frac{i \lambda}{\rho_{k}}\right) e^{\rho_{k}(i \lambda)}} .
$$

The remark which follows Theorem 2 will now be verified. Suppose first that $P\{x(T)>0\}=0$ for all $T>0$. It follows from (1.6) for $z=x+i y$ with $y<0$ that

$$
\left|e^{T \bar{\psi}(z)}\right| \leq \int_{-\infty}^{0} e^{-y \alpha} d_{\alpha} P\{x(T) \leq \alpha\} \leq 1
$$

which implies that $\Psi(z)$ can never take on a positive value for $\operatorname{Im} z<0$. Thus, if we know that $\Psi(z)=s>0$ has infinitely many (and hence at least one) solutions for $\operatorname{Im} z<0$, it follows that $P\{x(T)>0\}>0$ for some $T>0$. On the other hand, suppose $P\left\{x\left(T_{0}\right)>0\right\}>0$ for some fixed $T_{0}>0$. Then, the function $f(y)$ defined for $y<0$ by

$$
f(y)=\int_{-\infty}^{\infty} e^{-y \alpha} d_{\alpha} P\left\{x\left(T_{0}\right) \leq \alpha\right\}=e^{T \bar{\psi}(i y)}
$$


has a positive second derivative which is bounded away from zero. This means $f(y)=1$ for at most one negative $y$ and that $f(y)$ takes on just once all positive values for $y<0$. Moreover, $f(y)$ increases to plus infinity as $y$ decreases to minus infinity. By (5.7) $\psi(i y)$ is necessarily real-valued so that $\Psi(i y)=s>0$ has exactly one solution $y_{s}$ for $y<0$. What is more $\left|y_{s}\right| \rightarrow \infty$ as $s \rightarrow \infty$. Finally, if $\tilde{\rho}_{k}=\sigma_{k}+i \tau_{k}\left(\tau_{k}<0\right)$ and $\psi\left(\tilde{\rho}_{k}\right)=s>0$,

$$
e^{T_{0} \bar{\Psi}\left(i y_{s}\right)}=e^{T_{0} \bar{\Psi}\left(\tilde{\rho_{k}}\right)} \leq \int_{-\infty}^{\infty} e^{-\tau_{k} \alpha} d_{\alpha} P\left\{x\left(T_{0}\right) \leq \alpha\right\}=e^{T_{0} \bar{\psi}\left(i \tau_{k}\right)}
$$

which in turn yields $0<s=\Psi\left(i y_{s}\right) \leq \Psi\left(i \tau_{k}\right)$. Hence, $\left|y_{s}\right| \leq\left|\tilde{\rho}_{k}\right|$, i.e. $\rho_{1}=i y_{u}$, and the remark is verified.

\section{BIBLIOGRAPHY}

1. F. Spitzer, A combinatorial lemma and its application to probability theory, Trans. Amer. Math. Soc. vol. 82 (1956) pp. 323-339.

2. J. L. Doob, Stochastic processes, New York, Wiley, 1953.

3. L. V. Ahlfors, Complex analysis, New York, McGraw-Hill, 1953.

4. D. A. Darling, The maximum of sums of stable random variables, Trans. Amer. Math. Soc. vol. 83 (1956) pp. 164-169.

5. Sven Täcklind, Sur le risque de ruine dans des jeux inéquitables, Skandinavisk Aktuarietidskrift vol. 25 (1942) pp. 1-42.

6. G. Arfwedson, Research in collective risk theory, Part I, Skandinavisk Aktuarietidskrift (1954) Häft 3-4 pp. 191-223.

UNIVERSITY OF MiNNESOTA, MinNEAPOLIS, MinN. 